\title{
Exploring the somatic NF1 mutational spectrum associated with NF1 cutaneous neurofibromas
}

\author{
Laura Thomas ${ }^{1}$, Gill Spurlock ${ }^{1}$, Claire Eudall ${ }^{1}$, Nick S Thomas ${ }^{1}$, Matthew Mort ${ }^{1}$, Stephen E Hamby ${ }^{2}$, \\ Nadia Chuzhanova ${ }^{2}$, Hilde Brems ${ }^{3}$, Eric Legius ${ }^{3}$, David N Cooper ${ }^{1}$ and Meena Upadhyaya ${ }^{\star, 1}$
}

\begin{abstract}
Neurofibromatosis type-1 (NF1), caused by heterozygous inactivation of the NF1 tumour suppressor gene, is associated with the development of benign and malignant peripheral nerve sheath tumours (MPNSTs). Although numerous germline NF1 mutations have been identified, relatively few somatic NF1 mutations have been described in neurofibromas. Here we have screened 109 cutaneous neurofibromas, excised from 46 unrelated NF1 patients, for somatic NF1 mutations. NF1 mutation screening (involving loss-of-heterozygosity ( $\mathrm{LOH}$ ) analysis, multiplex ligation-dependent probe amplification and DNA sequencing) identified 77 somatic NF1 point mutations, of which 53 were novel. LOH spanning the NF1 gene region was evident in 25 neurofibromas, but in contrast to previous data from MPNSTs, it was absent at the TP53, CDKN2A and RB1 gene loci. Analysis of DNA/RNA from neurofibroma-derived Schwann cell cultures revealed NF1 mutations in four tumours whose presence had been overlooked in the tumour DNA. Bioinformatics analysis suggested that four of seven novel somatic NF1 missense mutations (p.A330T, p.Q519P, p.A776T, p.S1463F) could be of functional/clinical significance. Functional analysis confirmed this prediction for p.S1463F, located within the GTPase-activating protein-related domain, as this mutation resulted in a 150-fold increase in activated GTP-bound Ras. Comparison of the relative frequencies of the different types of somatic NF1 mutation observed with those of their previously reported germline counterparts revealed significant $(P=0.001)$ differences. Although non-identical somatic mutations involving either the same or adjacent nucleotides were identified in three pairs of tumours from the same patients $(P<0.0002)$, no association was noted between the type of germline and somatic $N F 1$ lesion within the same individual.
\end{abstract}

European Journal of Human Genetics (2012) 20, 411-419; doi:10.1038/ejhg.2011.207; published online 23 November 2011

Keywords: neurofibromatosis type 1; cutaneous neurofibromas; somatic and germline NF1 mutations

\section{INTRODUCTION}

Neurofibromatosis type-1 (NF1; MIM number 162200) is an autosomal dominant tumour predisposition syndrome, affecting about 1 in 4000 people worldwide. NF1 is associated with a highly variable clinical phenotype. ${ }^{1}$ It results from inactivating mutations in the 17q11.2-located NF1 gene that leads to the functional loss of its protein product, neurofibromin. The NF1 gene spans $\sim 282 \mathrm{~kb}$ of genomic DNA, contains 61 exons (four of which are alternatively spliced) and encodes a $\sim 9-\mathrm{kb}$ mRNA transcript. Neurofibromin is a highly conserved RAS-GTPase-activating protein (GAP) that is directly involved in the regulation of Ras signalling. ${ }^{2-4}$ It downregulates Ras activation in the cell, thereby also downregulating the multiple downstream effectors activated by Ras, including the $\mathrm{PI} 3 \mathrm{~K}$ and the mitogen-activated kinase signalling cascades, which are involved in regulating cellular proliferation, DNA synthesis and apoptosis.

NF1 is a classic tumour suppressor gene and, consistent with Knudson's 'two-hit hypothesis', all patients harbour both a normal and a dysfunctional NF1 gene copy, the latter containing the inherited (germline) mutation. Tumours arising in such patients contain a subpopulation of cells manifesting biallelic inactivation of the NF1 gene as a consequence of an acquired somatic NF1 mutation. Whereas the patient-associated NF1 germline mutational spectrum has been fairly well characterised (at least 1290 different NF1 gene mutations had been identified by August 2011; Human Gene Mutation Database (HGMD) $),{ }^{5}$ comparatively few somatic NF1 mutations have so far been identified in NF1-associated tumours. This paucity is mainly due to the inherent difficulty in detecting such somatic mutations as a consequence of the cellular heterogeneity of the tumour tissue, but is also a consequence of the relatively small number of benign NF1 tumours analysed to date. ${ }^{6}$

One of the most characteristic clinical features manifested by NF1 patients is the growth of benign peripheral nerve sheath tumours (neurofibromas) in the skin. Whereas cutaneous neurofibromas are present in almost all adult NF1 patients, ${ }^{7}$ plexiform neurofibromas (PNFs), a more diffuse type of tumour, are present in only $30-50 \%$ of patients. However, $10-15 \%$ of PNFs are transformed into malignant peripheral nerve sheath tumours (MPNSTs), a major cause of mortality in NF1. ${ }^{8}$ Cutaneous neurofibromas usually appear during adolescence, although they may occasionally occur at an earlier age. The marked variability in neurofibroma number frequently observed between affected individuals from the same NF1 family has led to the suggestion that modifying loci might also be involved in tumour development. ${ }^{9}$ The neurofibromas themselves exhibit extensive

${ }^{1}$ Institute of Medical Genetics, School of Medicine, Cardiff University, Cardiff, UK; ${ }^{2}$ School of Science and Technology, Nottingham Trent University, Nottingham, UK; ${ }^{3}$ Department of Human Genetics, Catholic University of Leuven, Herestraat, Belgium

*Correspondence: Professor M Upadhyaya, Institute of Medical Genetics, School of Medicine, Cardiff University, Heath Park Way, Cardiff CF14 4XN, UK. Tel: +44 2920 744081 ; Fax: +44 2920 746551; E-mail: upadhyaya@cardiff.ac.uk

Received 25 May 2011; revised 4 October 2011; accepted 6 October 2011; published online 23 November 2011 
cellular heterogeneity, being composed of hyperproliferative Schwann cells, fibroblasts, mast cells and perineural cells, but it is the Schwann cells specifically in which the NF1 gene becomes biallelically inactivated in cutaneous neurofibromas. ${ }^{10,11}$

Currently, our understanding of the biological mechanisms underlying NF1 tumourigenesis is rather limited, although recent studies in mouse have (i) confirmed a direct role for the tumour microenvironment and (ii) identified skin-derived precursor cells as the cell of origin for cutaneous neurofibromas. ${ }^{12}$ In studies of NF1 patients, somatic mutations affecting a number of other tumour suppressor genes, including TP53, CDKN2A and RB1 (variously involved in cell cycle regulation, DNA synthesis and apoptosis), have also been identified in MPNSTs, ${ }^{13-17}$ PNFs $^{18,19}$ and those cutaneous neurofibromas that have been removed from NF1 patients who carry a particularly high tumour burden. ${ }^{20}$

It is currently unclear (i) why some types of neurofibroma are present from birth, whereas others develop only during adolescence, (ii) what initiates neurofibroma growth and (iii) what determines the number of tumours that develop in a given individual. The acquisition of detailed information on the type, frequency and intragenic location of somatic mutations in the NF1 gene in a sizeable cohort of patients represents a first step towards improving our understanding of the molecular mechanisms underlying NF1 tumourigenesis. To this end, in the most comprehensive analysis of its kind performed to date, we have screened the NF1 gene both for loss-of-heterozygosity (LOH) and for microlesions in genomic DNA from 109 cutaneous neurofibromas derived from 46 unrelated patients.

\section{MATERIALS AND METHODS}

\section{Patient samples}

The study samples comprised a total of 109 cutaneous neurofibromas excised from 46 unrelated patients who displayed the requisite NIH clinical diagnostic criteria for NF1. ${ }^{21}$ These samples comprised 94 primary tumour samples taken from 43 patients, plus 15 Schwann cell lines cultured from individual neurofibromas excised from the remaining three patients. Primary tumour tissue was carefully macrodissected away from adjacent normal skin. DNA was then extracted from tumour tissue, cultured Schwann cell lines or patient lymphocytes using phenol/chloroform. ${ }^{11}$ This study was approved by the local research ethics committee. All patients provided their written informed consent.

\section{Schwann cell culture}

The 15 cutaneous neurofibromas used for primary Schwann cell culture were initially cut into two sections; one was used to culture $\mathrm{NF}^{-/-}$Schwann cells, whereas the other was used directly for somatic mutation analysis. Neurofibroma-derived Schwann cell samples were cultured as previously described. ${ }^{11,22,23}$ Immunocytochemistry was performed using a standard protocol. ${ }^{24}$

\section{Analysis of germline and somatic NF1 mutations}

DNA from all 94 tumour samples and 15 Schwann cell lines, together with samples of the corresponding patients' lymphocyte DNA, was initially screened for evidence of $\mathrm{LOH}$ using a panel of fluorescently tagged polymorphic markers (see below) encompassing the NF1 gene region (Figure 1). Those samples that were negative for LOH were then screened for (i) microlesions by DNA sequencing (Figure 1; see below) and (ii) intragenic NF1 deletions/duplications using MLPA (multiplex ligation-dependent probe amplification; see below; RefSeq: NM_000267). Direct sequencing of patient lymphocyte genomic DNA was also used to characterise the underlying germline NF1 mutations. Total RNA was extracted from Schwann cells. ${ }^{25}$ cDNA corresponding to the coding region of the NF1 gene was made by reverse transcription and PCR amplified in 24 overlapping fragments. ${ }^{25}$ Mutations identified at the cDNA level were always confirmed in genomic DNA by direct cycle sequencing. Details of the NF1 locus

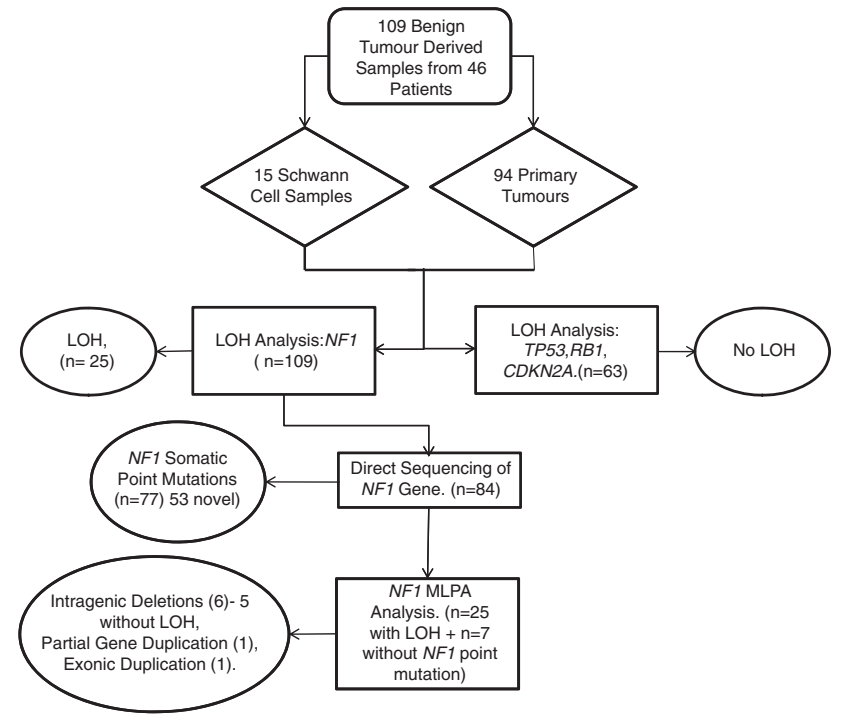

Figure 1 Work flow for detection of somatic mutations involving the NF1, TP53, RB1 and CDKN2A genes in 109 neurofibromas and neurofibromaderived Schwann cell lines.

LOH analysis, direct sequencing, NF1 MLPA analysis, LOH at TP53, RB1 and CDKN2A are given under the Supplementary methods.

\section{Functional analysis - site-directed mutagenesis}

The oligonucleotide primers for site-directed mutagenesis were designed individually according to the desired mutation using the QuickChange primer design programme (Agilent, Edinburgh, UK). PCR-based site-directed mutagenesis was performed using $10 \mathrm{ng} / \mu \mathrm{l}$ plasmid DNA, $125 \mathrm{ng}$ of each primer, and the following cycle conditions: $95^{\circ} \mathrm{C}, 30 \mathrm{~s} ; 55^{\circ} \mathrm{C}, 1 \mathrm{~min} ; 68^{\circ} \mathrm{C}, 8 \mathrm{~min}(18 \times$ cycles). A volume of $1 \mu \mathrm{l} D p n \mathrm{I}$ restriction enzyme (NEB; $10 \mathrm{U} / \mu \mathrm{l}$ ) was directly added to each reaction and incubated at $37^{\circ} \mathrm{C}$ for $1 \mathrm{~h}$. Plasmid DNA produced following site-directed mutagenesis was transfected into HEK293 cells using Lipofectamine 2000 (Invitrogen, Glasgow, UK) and following the manufacturers' protocol. Expression of the V5-tagged protein in untransfected controls and mutant cell lines was assessed by western blot.

\section{Ras ELISA}

The Ras activation ELISA was performed as described in the manufacturers' protocol (Millipore, Billerica, MA, USA, Cat number: 17-497). Following addition of the chemiluminescent substrate, the Ras ELISA was assessed using a microtitre plate luminometer (Applied Biosystems, Foster City, CA, USA). Relative luminescence (RLU) was evaluated for each sample using WINGLOW software (Perkin Elmer, Waltham, MA, USA) between 5 and 60 min after the substrate was added.

\section{Comparison of germline and somatic mutational spectra}

Data sets of somatic mutations (comprising 30 missense/nonsense mutations and 37 microdeletions/microinsertions) identified here and germline mutations (comprising 306 missense/nonsense mutations and 512 microdeletions/microinsertions derived from the HGMD (April 2011 release) $)^{5}$ in the NF1 gene were compared in this study. Sequences flanking each NF1 mutation (either somatic or germline) were screened for the presence of direct and inverted repeats and symmetric elements (of length $\geq 6 \mathrm{bp}$ and $\leq 20 \mathrm{bp}$ apart) by means of complexity analysis, ${ }^{26}$ to identify sequences capable of facilitating the formation of non-B DNA structures. Sequences flanking microdeletions and microinsertions were screened for the presence of polynucleotide runs of length $\geq 4 \mathrm{bp}$ known to represent mutational hotspots for duplications and 'de-duplications $^{27}$ via slipped mispairing at DNA replication. In addition, $\mathrm{C}>\mathrm{T}$ and $\mathrm{G}>\mathrm{A}$ substitutions within $\mathrm{CpG}$ and $\mathrm{CpHpG}$ oligonucleotides (where $\mathrm{H}$ is either $\mathrm{C}$, $\mathrm{A}$ or $\mathrm{T}$ ) were also recorded for both germline and somatic mutational spectra of 
missense and nonsense mutations. The similarities and differences of somatic and germline mutational spectra, with respect to the above features, were assessed by means of Fisher's exact test.

\section{Assessment of functionality or otherwise of identified missense mutations}

In an attempt to determine whether (and if so, how) the seven identified somatic NF1 missense mutations might disrupt neurofibromin structure/ function, these amino acid substitutions were analysed using a range of bioinformatic tools. The combined output of these tools was then used to predict which of these mutations is likely to be of functional/pathological significance. Evolutionary conservation of the mutated neurofibromin amino acid residues, across an alignment of 34 placental mammal species, was assessed using phyloP. ${ }^{28}$ phyloP assesses evolutionary conservation/acceleration using a likelihood ratio test (a positive phyloP score represents a conserved nucleotide, whereas a negative phyloP score indicates that the nucleotide has experienced faster evolution than expected under neutral drift). Protein structure/function disruption and/or the disease-causing potential of missense mutations was evaluated using five different tools: MutPred, ${ }^{29,30}$ SIFT, $^{31}$ PolyPhen $^{32}$ and Mutation Taster; ${ }^{33}$ the potential effect of the missense variants on exon skipping, via loss of exonic splicing enhancers (ESE) and/or gain of exonic splicing silencers (ESS), was ascertained using Skippy. ${ }^{34}$ Skippy outputs a log odds ratio score that quantifies the likelihood that a given nucleotide substitution may lead to exon skipping; a Skippy log odds ratio score threshold $>2$ was used to identify high-confidence variants that may result in exon skipping via ESE loss and/or ESS gain. The ESE and ESS motifs considered in this analysis (Supplementary Table 1) are derived from the NI-ESE and NI-ESS set of exonic regulatory elements. ${ }^{35}$ The NI-ESE and NI-ESS sets of exonic regulatory elements motifs have previously been identified as providing the strongest signal for identifying exon-skipping variants. ${ }^{34}$ A previously described, neural network was used to assess splice-site disruption and cryptic splice-site activation. ${ }^{36}$ The six different lines of evidence (evolutionary conservation (phyloP), MutPred, SIFT, PolyPhen2, Mutation Taster and the splicing predictions) were individually converted to a binary classification ( 0 or 1$)$, with a ' 1 ' representing deleterious (or 'conserved' in the case of phyloP) and a ' 0 ' representing either a tolerated, non-conserved or a putative passenger mutation. Giving each line of evidence an equal weighting, the majority class (' 0 ' or ' 1 ') for each mutation was used to assign the mutation as being either of functional significance (majority of ' 1 's) or not (majority of '0's), or unclassified (equal number of ' 1 's and '0's).

\section{RESULTS}

As a first step in this analysis, constitutional NF1 germline mutations were identified by means of MLPA and DNA sequencing in lymphocyte DNA from 38 of the 46 NF1 patients. This represents an $83 \%$ mutation detection rate, which is comparable to previously reported mutation rates for this gene, although it is still significantly lower than the mutation detection rate from the study by Messiaen et al (2000). ${ }^{37-39}$ These heterozygous inherited lesions comprised 36 truncating mutations and 2 missense mutations, of which 13 were novel (Table 1). All germline mutations were subsequently confirmed by identification in the corresponding tumour DNA samples. Eighteen of the identified NF1 germline mutations have been previously reported (Table 1, Supplementary Table 2), whereas six have been noted as somatic mutations in neurofibromas, either in this or earlier studies (Supplementary Tables 2 and 3). One germline, NF1 splice site mutation $(c .3113+1 \mathrm{G}>\mathrm{A})$, in this study has also been previously detected as a somatic mutation in a glomus tumour (Supplementary Tables 2 and 3).

A combination of LOH analysis, DNA sequencing and MLPA was used to identify a total of 77 somatic NF1 point mutations (53 novel) in genomic DNA from the 109 neurofibroma-derived samples (Table 1). These mutations were confirmed by sequencing both DNA strands. None of these lesions were evident in analyses of the corresponding patient lymphocyte DNA. The 77 somatic NF1 point mutations identified comprised 31 small (1-80 bp) deletions, 22 nonsense mutations, 9 splice site mutations, 8 missense mutations, 6 small (1-2 bp) insertions and an indel (insertion/deletion).

In 25 of the 109 neurofibroma-derived samples, LOH of the NF1 gene region was evident. In 4 of these 25 cases (in unrelated individuals), a deletion of the entire NF1 gene was present. The application of MLPA and LOH analysis to 24 of the 25 tumour samples did not reveal any intragenic rearrangement, suggesting that the NF1-LOH observed in 24 tumours was probably due to mitotic recombination. In the remaining case (T100), $\mathrm{LOH}$ was found to have resulted from the deletion of exons 38-49 of the NF1 gene (Table 1). Unfortunately, independent confirmation of the results of MPLA analysis by FISH could not be performed, because most of the available tumour samples had been frozen post excision.

In the remaining seven samples, without LOH or an NF1 point mutation, two single exon deletions (exon 8 and exon 16, respectively), two intragenic duplications (exon 8 and exons 19b-25, respectively) and three large (type 1) genomic deletions (Table 1) were identified. ${ }^{40}$

Analysis of DNA and RNA from neurofibroma-derived Schwann cell cultures allowed us to detect the somatic mutations in four tumours in which the somatic mutation had not been identified by sequence analysis of the original tumour-derived DNA sample. Thus, for example, the somatic NF1 frameshift mutation (c.1888delG, p.V630fsX), identified in tumour T89.1, was only detectable in DNA from cultured Schwann cells and was not evident in the original tumour DNA sample (Figure 2).

Although seven novel somatic NF1 missense mutations (A330T, H393D, H393L, Q519P, G629R, A776T, S1463F) were identified here, their pathological relevance was uncertain. None of these substitutions had been noted in any of our previous NF1 gene mutation screens (involving chromosome 17 from at least 1000 individuals), and all involved amino acid residues that were evolutionarily highly conserved (Table 2). To evaluate the potential functionality of these seven novel missense mutations, we used a suite of bioinformatic tools to assess their impact on protein structure, function and mRNA splicing (Table 2 and Supplementary Table 1). Taking the results together, we surmise that at least four of the seven missense mutations (p.A330T, p.Q519P, p.A776T, p.S1463F) could be of functional/clinical significance (Table 2 and Supplementary Table 1). Two of these missense mutations (p.A330T and pS1463F) were predicted to exert their influence via disruption of protein structure/function (Supplementary Table 1). In addition, the nucleotide substitution underlying p.A776T, located in the first base of exon 15, was predicted to result in the weakening of the splice site (Supplementary Table 1). We then tested our predictions, as far as we could, by performing functional analysis on the p.S1463F missense mutation. This was possible because of its location within the GAP-related domain, as an assay for GAP activity is available. Transfection of cell lines with the p.S1463F mutation resulted in a 150-fold increase in activated GTP-bound Ras (by comparison with cell lines carrying the wild-type NF1 protein) as evidenced by the level of fluorescence recorded by the luminometer $\left(4.5 \times 10^{6} \mathrm{RLU}\right.$; Supplementary Figure 1). Therefore, at least in the case of this GAP-related domain mutation, the functional assay confirmed the bioinformatic predictions.

To explore whether the type of somatic mutation identified in each cutaneous neurofibroma was independent of the type of mutation observed in the germline in the same tumour, the specific combinations of germline and somatic NF1 mutations identified in the 62 matched lymphocyte/tumour pairs (i.e., where matching germline and somatic mutation data were available) were examined (Supplementary 


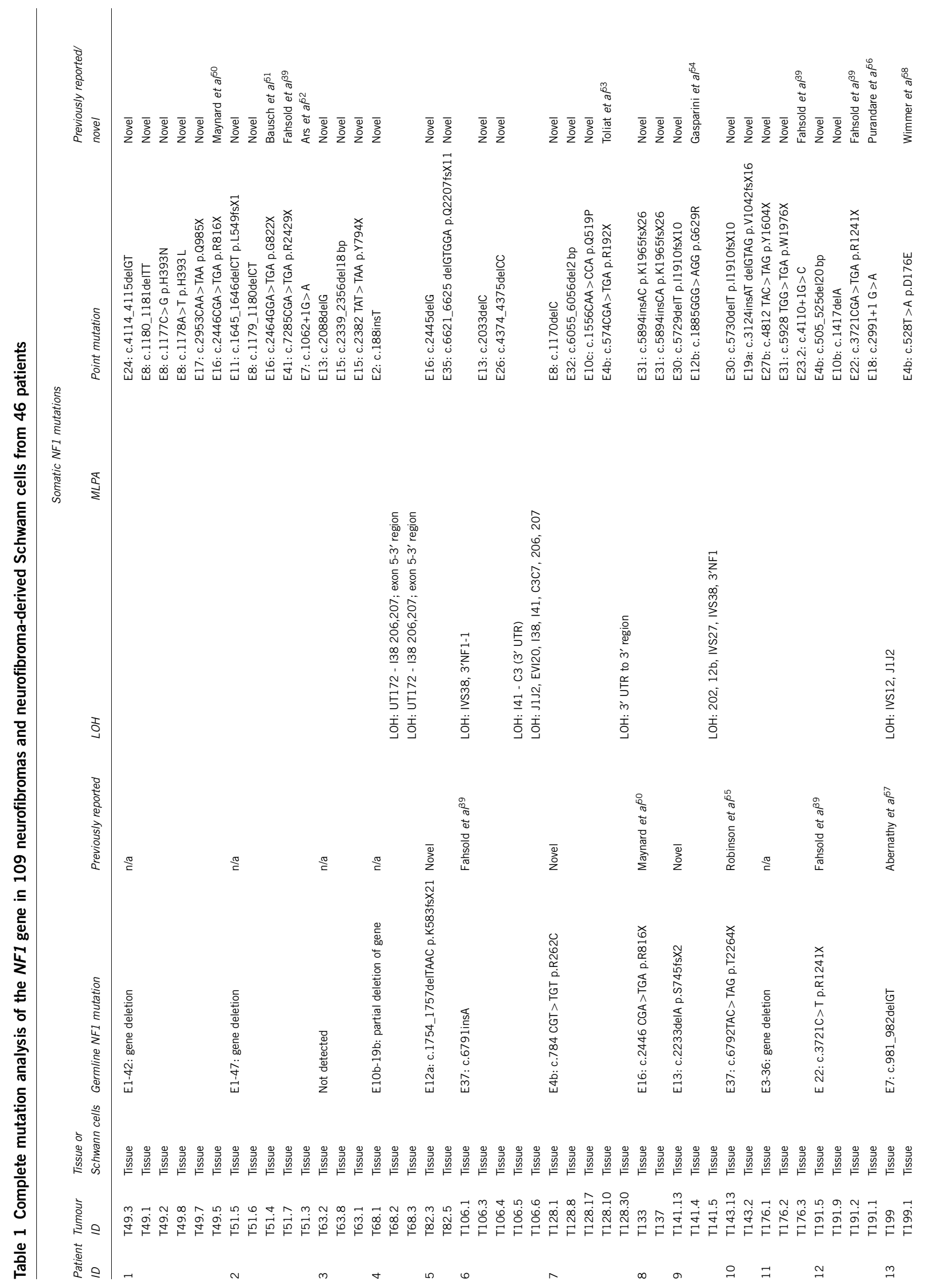




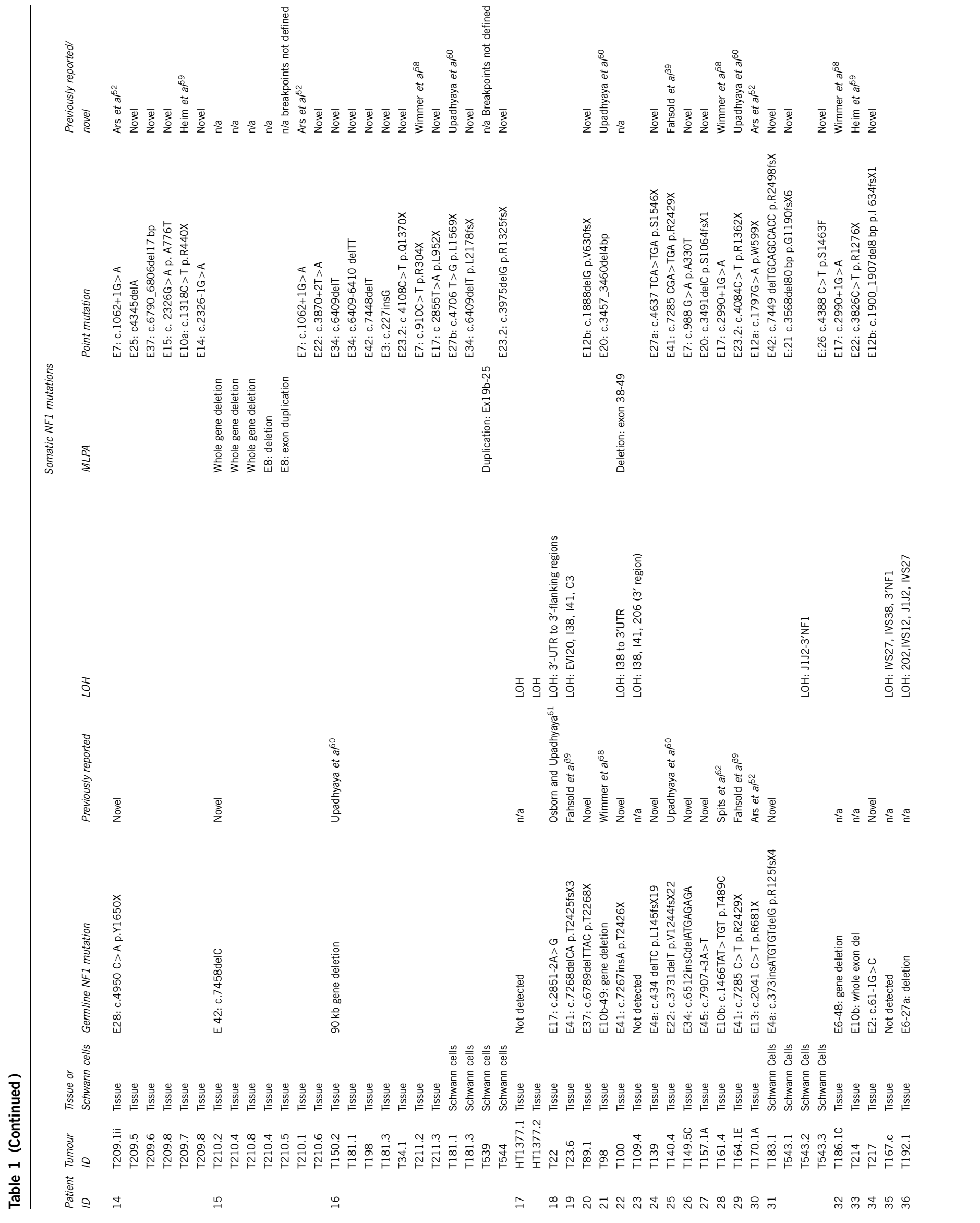




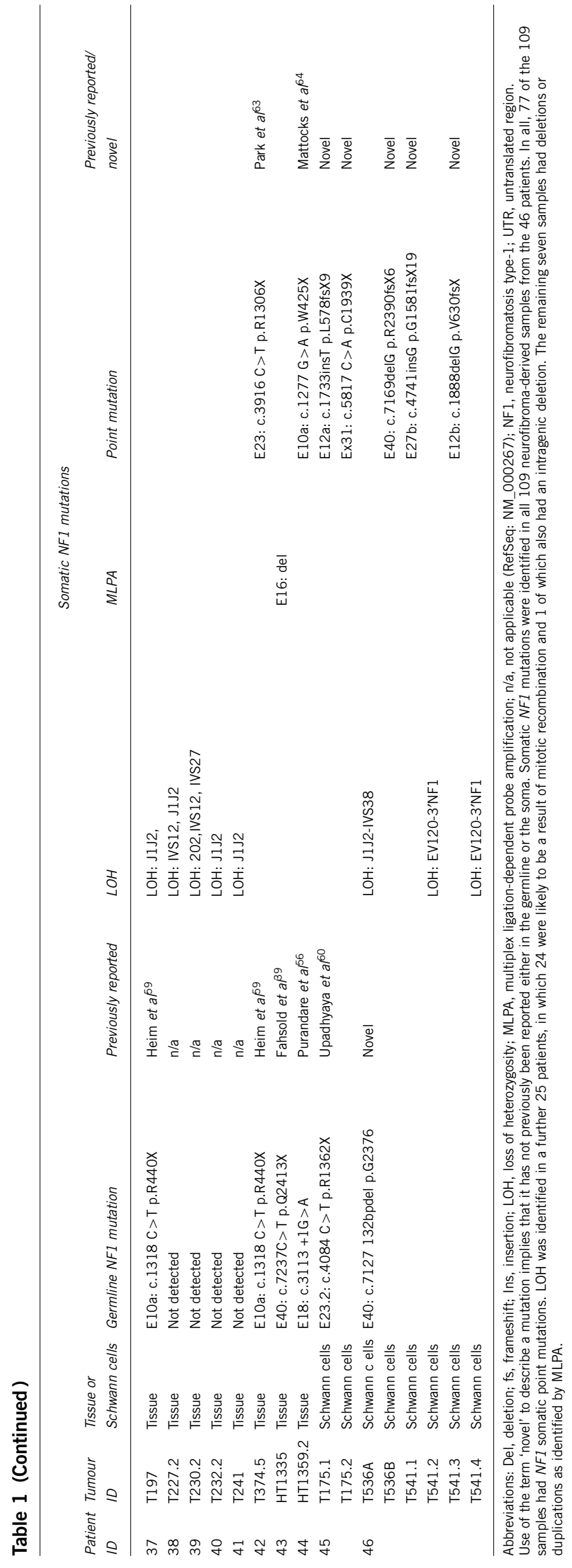

Table 4). The type of somatic mutation was found to be independent $(P=0.684)$ of the type of germline mutation (and vice versa) in the same tumour when somatic missense, nonsense/frameshift mutations and microdeletions/microinsertions, as well as gross deletions and nonsense/frameshift germline mutations were considered.

Comparison of the relative frequencies of observed somatic NF1 missense and nonsense mutations, microdeletions, microinsertions and splicing mutations with the frequencies of their NF1 counterparts in the germline (as reported in HGMD) 5 indicated significant (Fisher's exact test, two-tailed $P=0.001$ ) differences. Indeed, inspection of the relative mutation frequencies revealed significantly greater proportions of somatic NF1 nonsense mutations (29\%) and microdeletions (41\%), and smaller proportions of NF1 splicing mutations (12\%) and microinsertions $(8 \%)$ as compared with their germline equivalents $(16,31,26$ and $15 \%$, respectively). The proportion of somatic $\mathrm{C}>\mathrm{T}$ and $\mathrm{G}>\mathrm{A}$ missense/nonsense mutations located within $\mathrm{CpG}$ and $\mathrm{CpHpG}$ was found to be significantly (Fisher's exact test, two-tailed $P=0.004)$ higher (42\%) than the corresponding proportion of known NF1 germline mutations (19\%). Somatic microdeletions and microinsertions ( 31 and 6 , respectively) were found to occur in the vicinity of symmetric repeats more frequently than was the case for the germline NF1 mutations (Fisher's exact test, one-tailed $P=0.019$ ). In a similar vein, somatic NF1 missense/nonsense mutations were found to exhibit a tendency to occur in the vicinity of inverted repeats more frequently than in the germline $(P=0.055)$. Examples of sequence features that could have mediated NF1 germline and somatic mutations are depicted in Supplementary Figure 2.

No evidence for LOH involving the TP53, CDKN2A and RB1 gene regions was found in any of the 109 tumour and Schwann cell DNA samples.

\section{DISCUSSION}

The biological significance of specific somatic NF1 gene mutations for the tumourigenic process and the possible interplay with their germline counterparts have been difficult issues to address, owing to the paucity of somatic mutational data from neurofibromas and MPNSTs. The main aim of this study was to use a large panel of cutaneous neurofibromas $(N=109)$ from $46 \mathrm{NF1}$ patients to improve the definition of the somatic NF1 mutational spectrum.

In all, 53 of the 77 characterised somatic NF1 mutations identified in this study of cutaneous neurofibromas were novel, having not been previously reported as either germline or somatic lesions ( 13 of the 38 germline NF1 mutations detected in the $46 \mathrm{NF} 1$ patients were also novel (see Table 1)).

Schwann cell analysis is known to increase the efficiency of somatic mutation detection, ${ }^{12}$ and this was certainly found to be the case in the present study (Figure 2). Unfortunately, the culture of Schwann cells from multiple tumours is a labour-intensive procedure.

The cutaneous neurofibroma-associated somatic NF1 mutational spectrum characterised in this study appears broadly similar in its distribution of frameshift, nonsense, missense and splice site mutations to those mutational spectra previously reported in the context of PNFs and MPNSTs. ${ }^{17,18}$ Indeed, three of the somatic NF1 mutations characterised here (p.R816X, p.R304X, p.L1569X) have previously been identified as somatic lesions (two of these three mutations are CpG located and therefore compatible with a mechanism of methylation-mediated deamination of 5-methylcytosine) in both benign and malignant tumours. ${ }^{41-46}$

A total of seven novel somatic NF1 missense mutations were identified during the course of this study. To ascertain their functional/pathological relevance, we used a series of bioinformatic tools 


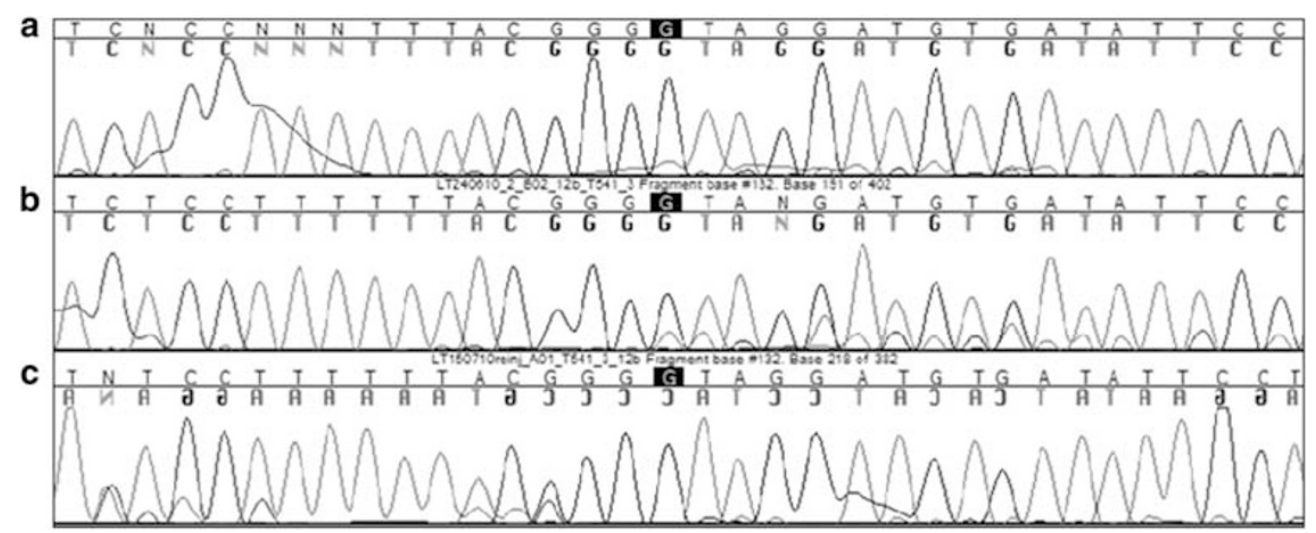

Figure 2 DNA sequence trace of the novel somatic heterozygous frameshift mutation (c.1888delG p.V630fsX) detected in exon 37 of the NF1 gene from patient T89.1. (a) 5'-3' sequence from neurofibroma tumour tissue, and (b) $5^{\prime}-3^{\prime}$ sequence from Schwann cells cultured from the same neurofibroma. (c) $3^{\prime}-5^{\prime}$ sequence from Schwann cells cultured from the same neurofibroma, illustrating that the G deletion was only detectable in the DNA trace from cultured Schwann cells.

Table 2 Bioinformatic assessment of protein structure/function disruption for the eight identified somatic NF1 missense mutations

\begin{tabular}{|c|c|c|c|c|c|c|}
\hline $\begin{array}{l}\text { Nucleotide } \\
\text { substitution }\end{array}$ & $\begin{array}{l}\text { Amino-acid } \\
\text { change }\end{array}$ & $\begin{array}{l}\text { Evolutionary } \\
\text { conservation } \\
\text { (phyloP) }\end{array}$ & $\begin{array}{l}\text { MutPred general score } \\
\text { (probability of deleterious } \\
\text { mutation) }\end{array}$ & SIFT prediction & PolyPhen2 & Mutation taster \\
\hline c. $988 \mathrm{G}>\mathrm{A}$ & p.A330T & Conserved (2.5) & Deleterious (0.56) & AFFECTS PROTEIN FUNCTION (0.01) & Probably damaging $(0.95)$ & Disease causing $(0.91)$ \\
\hline c. $1177 C>G$ & p.H393D & Conserved (2.5) & Non-deleterious (0.41) & TOLERATED $(0.20)$ & Benign (0.05) & Disease causing $(0.78)$ \\
\hline c. $1178 \mathrm{~A}>\mathrm{T}$ & p.H393 L & Conserved (2.0) & Non-deleterious (0.28) & TOLERATED (1.00) & Benign (0.03) & Disease causing $(0.88)$ \\
\hline c. $1556 \mathrm{~A}>\mathrm{C}$ & p.Q519P & Conserved (0.9) & Non-deleterious (0.16) & TOLERATED (2.20) & Possibly damaging $(0.24)$ & Disease causing $(0.95)$ \\
\hline c. $1885 \mathrm{G}>\mathrm{A}$ & p.G629R & Conserved (2.5) & Deleterious (0.90) & TOLERATED $(0.63)$ & Benign (0.00) & Polymorphism \\
\hline c. $2326 \mathrm{G}>\mathrm{A}$ & p.A776T & Conserved (2.4) & Deleterious (0.65) & AFFECTS PROTEIN FUNCTION (0.05) & Probably damaging (0.94) & Disease causing (0.99) \\
\hline c. $4388 \mathrm{C}>\mathrm{T}$ & p.S1463F & Conserved (2.8) & Non-deleterious (0.46) & AFFECTS PROTEIN FUNCTION (0.04) & Possibly damaging (0.59) & Disease causing (0.99) \\
\hline
\end{tabular}

Abbreviation: NF1, neurofibromatosis type-1.

to assess a number of different parameters including the evolutionary conservation of the affected residues, as well as the potential effect of the mutations on protein structure, function and mRNA splicing. We concluded that at least four of the seven somatic missense mutations (p.A330T, p.Q519P, p.A776T, p.S1463F) could be of functional/clinical significance. To test our predictions, we performed a functional analysis on the only missense mutation located in the GAP-related domain of neurofibromin (p.S1463F). By comparison with cell lines carrying the wild-type NF1 protein, transfection of cell lines with the p.S1463F mutation led to a 150-fold increase in activated GTP-bound Ras. This concurred with our prediction that p.S1463F was likely to be of functional/pathological importance.

The identification of NF1-associated LOH (probably associated with mitotic recombination) in 24 tumours is consistent with the results reported in a recent study of neurofibromas that assessed NF1associated LOH in this type of tumour. ${ }^{47}$ In our study, MLPA analysis succeeded in identifying only a single intragenic deletion in one neurofibroma, indicating that mitotic recombination was the likely mechanism for $\mathrm{LOH}$ in the remaining 24 neurofibromas. Our previous studies found significantly higher levels (approximately 70 and $90 \%$, respectively) of NF1-associated LOH in both PNFs and MPNSTs, ${ }^{17,18}$ an indication that NF1-LOH may be less prominent in benign neurofibromas than in PNFs and malignant tumours. One potential confounding factor here is cellular heterogeneity, but we do not consider that this would have substantially hampered the detection of intragenic deletions and duplications by MLPA in this study. Tumour-associated microsatellite instability was not analysed here, as our previous studies failed to detect significant levels of microsatellite instability in these cutaneous neurofibromas. ${ }^{44}$

Although LOH within the NF1 gene region was observed in 25 cutaneous neurofibromas, we sought for the remaining intragenic somatic lesions to ascertain whether there might be a relationship between the type of somatic NF1 mutation found in a given tumour and the type of germline NF1 mutation in the same patient. Analysis of the somatic NF1 mutation data (Table 1) indicated that, with the exception of six tumours derived from three unrelated patients (T49.2/T49.3; T133.2/T137; and T150.2/T180.1), all individual cutaneous neurofibromas derived from the same patient were found to harbour independent somatic NF1 mutations, consistent with the findings of previous studies. ${ }^{11,20,25}$ Interestingly, the somatic mutations identified in the patient-paired tumours T49.2/T49.8, T133/T137 and T150.2/T181.1 involve either the same or adjacent nucleotides within the same codons. Thus, for example, neurofibromas T49.2 and T49.8 harbour c.1177C $>\mathrm{G}$ and c.1178A $>\mathrm{T}$ substitutions, respectively, both involving codon His393. Neurofibromas T133/T137 (c.5894insAC and c.5894insCA) and T150.2/T181.1 (c.6409delT and c.6409delTT) constitute similar examples. The probability of observing mutations in two consecutive nucleotides by chance alone, assuming that the mutation rate $(\lambda)$ is constant across the NF1 gene, was estimated by means of a Poisson distribution, with $\lambda$ defined as the density of observed somatic mutations (both single base-pair substitutions and microdeletions/microinsertions) per nucleotide, and equals $(30+37) / 8457=0.008\left(1.26 \times 10^{-4}\right)$. We estimate that the probability of observing three such events in an analysis 
of the 125 possible pairwise comparisons of pairs of tumours derived from the 17 patients who donated multiple tumours to this study is $<0.0002$. Several repetitive elements were found in the vicinity of these juxtaposed mutations: for example, an inverted repeat and a symmetric element were found in the vicinity of the c.1177-8 mutations; a direct repeat and run of identical nucleotides span the site of the c.5894 mutations, whereas c.6409 occurs in the vicinity of symmetric elements. These repeat sequences may serve to facilitate the formation of multiple non-B DNA structures, ${ }^{48}$ thereby accounting for the hypermutability of these sites (shaded tumour IDs in Table 1). Previous mutation studies have also purportedly found evidence for potential clustering of mutations identified in separate neurofibromas from the same patient, ${ }^{48,49}$ but such conclusions have never before received formal statistical support. Although it is possible that the germline NF1 mutation might influence the location of subsequent somatic NF1 mutations, our current study on a relatively small number of paired germline somatic mutations have provided no evidence to support this postulate.

Although our previous study ${ }^{20}$ found evidence for $\mathrm{LOH}$ involving these three loci in cutaneous neurofibromas (albeit infrequently), such LOH was only identified in neurofibromas derived from NF1 patients presenting with very large numbers of such tumours. Only one of the patients in the present study had more than 500 cutaneous neurofibromas, and LOH analysis of his tumours (T210.1-T210.8, Table 1) failed to find any obvious changes at the TP53, CDKN2A and RB1 loci.

In summary, this is the first study to provide comprehensive (not merely LOH) NF1 mutational data from a large cohort of cutaneous neurofibromas. We identified 53 novel NF1 somatic mutations, which represent a considerable expansion of the known neurofibromaassociated NF1 somatic mutation spectrum. The results of this study highlight the importance of compiling large data sets of paired somatic and germline mutations, which should eventually help us both to understand the genetic pathways affected in NF1 tumourigenesis and the possible interactions between the germline and somatic NF1 gene lesions.

\section{CONFLICT OF INTEREST}

The authors declare no conflict of interest.

\section{ACKNOWLEDGEMENTS}

We are grateful to all our patients who participated in this study and who generously donated cutaneous neurofibroma material. We thank CRUK for their financial support. We would also thank Elisa Majounie for her assistance with Schwann cell culture, and the referring clinicians for their support.

1 Huson S: The neurofibromatoses: classification, clinical features and genetic counseling; in Kaufmann D (ed): Neurofibromatoses (Monographs in Human Genetics), 1st edn Switzerland: S Karger AG, 2008, Vol 16, pp 1-20.

2 Cichowski K, Jacks T: NF1 tumor suppressor gene function: narrowing the GAP. Cell 2001; 104: 593-604.

3 Arun D, Gutmann DH: Recent advances in neurofibromatosis type 1. Curr Opin Neurol 2004; 17: 101-105.

4 Gottfried ON, Viskochil DH, Fults DW, Couldwell WT: Molecular, genetic, and cellular pathogenesis of neurofibromas and surgical implications. Neurosurgery 2006; 58: 1-16. 5 Human Gene Mutation Database (HGMD) can be accessed at http://www.hgmd.org.

6 Bennett E, Thomas N, Upadhyaya M: Neurofibromatosis type 1: its association with the Ras/MAPK pathway syndromes. J Pediatr Neurol 2009; 7: 105-115.

7 Upadhyaya M, Huson SM, Davies M et al: An absence of cutaneous neurofibromas associated with a 3-bp inframe deletion in exon 17 of the NF1 gene (c.2970-2972 delAAT): evidence of a clinically significant NF1 genotype-phenotype correlation. Am J Hum Genet 2007; 80: 140-151.

8 Upadhyaya M: Genetic basis of tumorigenesis in NF1 malignant peripheral nerve sheath tumors. Front Biosci 2011; 16: 937-951.
9 Sabbagh A, Pasmant E, Laurendeau I et al: Unravelling the genetic basis of variable clinical expression in neurofibromatosis 1. Hum Mol Genet 2009; 18: 2768-2778.

10 Serra E, Rosenbaum T, Winner U et al: Schwann cells harbor the somatic NF1 mutation in neurofibromas: evidence of two different Schwann cell subpopulations. Hum Mol Genet 2000; 9: 3055-3064.

11 Maertens $\mathrm{O}$, Brems $\mathrm{H}$, Vandesompele $\mathrm{J}$ et al: Comprehensive NF1 screening on cultured Schwann cells from neurofibromas. Hum Mutat 2006; 27: 1030-1040.

12 Le LQ, Shipman T, Burns DK, Parada LF: Cell of origin and microenvironment contribution for NF1-associated dermal neurofibromas. Cell Stem Cell 2009; 4: 453-463.

13 Kourea HP, Orlow I, Scheithauer BW, Cordon-Cardo C, Woodruff JM: Deletions of the INK4A gene occur in malignant peripheral nerve sheath tumors but not in neurofibromas. Am J Pathol 1999; 155: 1855-1860.

14 Nielsen GP, Stemmer-Rachamimov AO, Ino Y, Moller MB, Rosenberg AE, Louis DN: Malignant transformation of neurofibromas in neurofibromatosis 1 is associated with CDKN2A/p16 inactivation. Am J Pathol 1999; 155: 1879-1884.

15 Mawrin C, Kirches E, Boltze C, Dietzmann K, Roessner A, Schneider-Stock R: Immunohistochemical and molecular analysis of p53, RB, and PTEN in malignant peripheral nerve sheath tumors. Virchows Arch 2002; 440: 610-615.

16 Mantripragada KK, Spurlock G, Kluwe L et al: High-resolution DNA copy number profiling of malignant peripheral nerve sheath tumors using targeted microarray-based comparative genomic hybridization. Clin Cancer Res 2008; 14: 1015-1024.

17 Upadhyaya M, Kluwe L, Spurlock G et al: The germline and somatic NF1 gene mutation spectrum in NF1-associated malignant peripheral nerve sheath tumours (MPNST). Hum Mut 2008; 29: 74-82.

18 Upadhyaya M, Spurlock G, Monem B et al: Germline and somatic NF1 gene mutations in plexiform neurofibromas. Hum Mutat 2008; 29: E112-E122.

19 Stewart $\mathrm{H}$, Bowker C, Edees S et al: Congenital disseminated neurofibromatosis type 1: A clinical and molecular case report. Am J Med Genet 2008; 146A: 1444-1452.

20 Thomas L, Kluwe L, Mautner V, Chuzhanova NA, Upadhyaya M: Analysis of somatic mutations in cutaneous neurofibromas from patients with high tumor burden. Neurogenetics 2010; 11: 391-400.

21 DeBella K, Szudek J, Friedman JM: Use of the National Institutes of Health criteria for diagnosis of neurofibromatosis 1 in children. Pediatrics 2000; 105: 608-614.

22 Rosenbaum T, Rosenbaum C, Winner U, Muller HW, Lenard HG, Hanemann CO: Long-term culture and characterization of human neurofibroma-derived Schwann cells. J Neurosci Res 2000; 61: 524-532.

23 Bottenstein JE, Sato GH: Growth of a rat neuroblastoma cell line in serum-free supplemented medium. Proc Natl Acad Sci USA 1979; 76: 514-517.

24 Bartley AN, Ross DW: Validation of p53 Immunohistochemistry as a prognostic factor in breast cancer in clinical practice. Arch Pathol Lab Med 2002; 126: 456-458.

25 Upadhyaya M, Han S, Consoli C et al: Characterization of the somatic mutational spectrum of the neurofibromatosis type 1 (NF1) gene in neurofibromatosis patients with benign and malignant tumors. Hum Mutat 2004; 23: 134-146.

26 Gusev VD, Nemytikova LA, Chuzhanova NA: On the complexity measures of genetic sequences. Bioinformatics 1999; 15: 994-999.

27 Kondrashov AS, Rogozin IB: Context of deletions and insertions in human coding sequences. Hum Mutat 2004; 23: 177-185.

28 Pollard KS, Hubisz MJ, Rosenbloom KR, Siepel A: Detection of nonneutral substitution rates on mammalian phylogenies. Genome Res 2010; 20: 110-121.

29 Li B, Krishnan VG, Mort ME et al: Automated inference of molecular mechanisms of disease from amino acid substitutions. Bioinformatics 2009; 25: 2744-2750.

30 Mort M, Evani US, Krishnan VG et al: In silico functional profiling of human diseaseassociated and polymorphic amino acid substitutions. Hum Mutat 2010; 31: 335-346.

$31 \mathrm{Ng}$ PC, Henikoff S: Predicting deleterious amino acid substitutions. Genome Res 2001; 11: 863-874.

32 Adzhubei IA, Schmidt S, Peshkin L et al: A method and server for predicting damaging missense mutations. Nat Methods 2008; 7: 248-249.

33 Schwarz JM, Rödelsperger C, Schuelke M, Seelow D: Mutation Taster evaluates disease-causing potential of sequence alterations. Nat Methods 2010; 7: 575-576.

34 Woolfe A, Mullikin JC, Elnitski L: Genomic features defining exonic variants that modulate splicing. Genome Biol 2010; 11: R20.

35 Stadler MB, Shomron N, Yeo GW, Schneider A, Xiao X, Burge CB: Inference of splicing regulatory activities by sequence neighborhood analysis. PLoS Genet 2006; 2: e191.

36 Krawczak M, Thomas NS, Hundrieser B et al: Single base-pair substitutions in exonintron junctions of human genes: nature, distribution, and consequences for mRNA splicing. Hum Mutat 2007; 28: 150-158.

37 Griffiths S, Thompson P, Frayling I, Upadhyaya M: Molecular diagnosis of neurofibromatosis type 1: 2 years experience. Fam Cancer 2007; 6: 21-34.

38 Messiaen LM, Callens T, Mortier G et al: Exhaustive mutation analysis of the NF1 gene allows identification of $95 \%$ of mutations and reveals a high frequency of unusual splicing defects. Hum Mutat 2000; 15: 541-555.

39 Fahsold R, Hoffmeyer S, Mischung $\mathrm{C}$ et al: Minor lesion mutational spectrum of the entire NF1 gene does not explain its high mutability but points to a functional domain upstream of the GAP-related domain. Am J Hum Genet 2000; 66: 790-818.

40 Dorschner MO, Sybert VP, Weaver M, Pletcher BA, Stephens K: NF1 microdeletion breakpoints are clustered at flanking repetitive sequences. Hum Mol Genet 2000; 9: 35-46.

41 Cooper DN, Mort M, Stenson PD, Ball EV, Chuzhanova NA: Methylation-mediated deamination of 5-methylcytosine appears to give rise to mutations causing human inherited disease in $\mathrm{CpNpG}$ trinucleotides as well as in $\mathrm{CpG}$ dinucleotides. Hum Genomics 2010; 4: 406-410.

42 Rodenhiser DI, Coulter-Mackie MB, Singh SM: Evidence of DNA methylation in the neurofibromatosis type 1 (NF1) gene region of 17q11.2. Hum Mol Genet 1993; 2: 439-444. 
43 Horan MP, Cooper DN, Upadhyaya M: Hypermethylation of the neurofibromatosis type 1 (NF1) gene promoter is not a common event in the inactivation of the NF1 gene in NF1-specific tumours. Hum Genet 2000; 107: 33-39.

44 Luijten M, Redeker S, van Noesel MM, Troost D, Westerveld A, Hulsebos TJ: Microsatellite instability and promoter methylation as possible causes of NF1 gene inactivation in neurofibromas. Eur J Hum Genet 2000; 8: 939-945.

45 Fishbein L, Eady B, Sanek N, Muir D, Wallace MR: Analysis of somatic NF1 promoter methylation in plexiform neurofibromas and Schwann cells. Cancer Genet Cytogenet 2005; 157: 181-186.

46 Maunakea AK, Nagarajan RP, Bilenky $M$ et al: Conserved role of intragenic DNA methylation in regulating alternative promoters. Nature 2010; 466: 253-257.

47 Garcia-Linares C, Fernández-Rodríguez J, Terribas E et al: Dissecting loss of heterozygosity (LOH) in neurofibromatosis type 1-associated neurofibromas: importance of copy neutral LOH. Hum Mutat 2011; 32: 78-90.

48 Bacolla A, Wang G, Jain A et al: Non-B DNA-forming sequences and WRN deficiency independently increase the frequency of base substitution in human cells. $J$ Biol Chem 2011; 286: 10017-10026.

49 Wiest V, Eisenbarth I, Schmegner C, Krone W, Assum G: Somatic NF1 mutation spectra in a family with neurofibromatosis type 1 : toward a theory of genetic modifiers. Hum Mutat 2003; 22: 423-427.

50 Maynard J, Krawczak M, Upadhyaya M: Characterization and significance of nine novel mutations in exon 16 of the neurofibromatosis type 1 (NF1) gene. Hum Genet 1997; 99: 674-676.

51 Bausch B, Borozdin W, Mautner VF et al: Germline NF1 mutational spectra and loss-ofheterozygosity analyses in patients with pheochromocytoma and neurofibromatosis type 1. J Clin Endocrinol Metab 2007; 92: 2784-2792.

52 Ars $\mathrm{E}$, Kruyer $\mathrm{H}$, Morell $\mathrm{M}$ et al: Recurrent mutations in the NF1 gene are common among neurofibromatosis type 1 patients. J Med Genet 2003; 40: e82.

53 Toliat MR, Erdogan F, Gewies A et al: Analysis of the NF1 gene by temperature gradient gel electrophoresis reveals a high incidence of mutations in exon 4b. Electrophoresis 2000; 21: 541-544.
54 Gasparini P, D’Agruma L, Pio de Cillis G, Balestrazzi P, Mingarelli R, Zelante L: Scanning the first part of the neurofibromatosis type 1 gene by RNA-SSCP: identification of three novel mutations and of two new polymorphisms. Hum Genet 1996; 97: 492-495.

55 Robinson PN, Böddrich A, Peters $\mathrm{H}$ et al: Hum Genet. Two recurrent nonsense mutations and a 4 bp deletion in a quasi-symmetric element in exon 37 of the NF1 gene. Hum Genet 1995; 96: 95-98.

56 Purandare SM, Lanyon WG, Connor JM: Characterisation of inherited and sporadic mutations in neurofibromatosis type-1. Hum Mol Genet 1994; 3: 1109-1115.

57 Abernathy CR, Rasmussen SA, Stalker HJ et al: NF1 mutation analysis using a combined heteroduplex/SSCP approach. Hum Mutat 1997; 9: 548-554.

58 Wimmer K, Roca X, Beiglböck H et al: Extensive in silico analysis of NF1 splicing defects uncovers determinants for splicing outcome upon $5^{\prime}$ splice-site disruption. Hum Mutat 2007; 28: 599-612.

59 Heim RA, Silverman LM, Farber RA, Kam-Morgan LN, Luce MC: Screening for truncated NF1 proteins. Nat Genet 1994; 8: 218-219.

60 Upadhyaya M, Osborn MJ, Maynard J, Kim MR, Tamanoi F, Cooper DN: Mutational and functional analysis of the neurofibromatosis type 1 (NF1) gene. Hum Genet 1997; 99: 88-92.

61 Osborn MJ, Upadhyaya M: Evaluation of the protein truncation test and mutation detection in the NF1 gene: mutational analysis of 15 known and 40 unknown mutations. Hum Genet 1999; 105: 327-332.

62 Spits C, De Rycke M, Van Ranst N et al: Preimplantation genetic diagnosis for neurofibromatosis type 1. Mol Hum Reprod 2005; 11: 381-387.

63 Park VM, Pivnick EK: Neurofibromatosis type 1 (NF1): a protein truncation assay yielding identification of mutations in $73 \%$ of patients. J Med Genet 1998; 35: 813820.

64 Mattocks C, Baralle D, Tarpey P, ffrench-Constant C, Bobrow M, Whittaker J: Automated comparative sequence analysis identifies mutations in $89 \%$ of NF1 patients and confirms a mutation cluster in exons 11-17 distinct from the GAP related domain. J Med Genet 2004; 41: e48.

Supplementary Information accompanies the paper on European Journal of Human Genetics website (http://www.nature.com/ejhg) 